\title{
Fried Rice Syndrome, a Disease of Fast World: Scientific Analysis
}

\author{
Mohammad Nazrul Islam \\ Department of Unani Medicine, Hamdard University Bangladesh, Bangladesh \\ *Corresponding author: Mohammad Nazrul Islam, Department of Unani Medicine; Hamdard University Bangladesh. \\ To Cite This Article: Mohammad Nazrul Islam. Fried Rice Syndrome, a Disease of Fast World: Scientific Analysis. Am J Biomed Sci \& Res. 2019 - 5(6). \\ AJBSR.MS.ID.000979. DOI: 10.34297/AJBSR.2019.05.000979.
}

Received: 紫September 30, 2019; Published: 眥 October 29, 2019

\begin{abstract}
World is changing and changing for the demand of the civilization. Peoples are used to prefer food from outside due to time constrain. They are used to take food which can be prepared easily and take less time to consume. That's why, Fried Rice menu is preferable than others menu. Peoples who are used to take Fried Rice in their regular meal, they have more chance to develop Fried Rice Syndrome than others. It is a food borne bacterial in-toxication which is caused by toxin of Bacillus cereus. To increase awareness among the consumer, food handler and food maker is the effective method to control the life-threatening food borne intoxication in the world.
\end{abstract}

\section{Introduction}

Fried Rice Syndrome is a food borne disease due to food intoxication by Bacillus cereus, a Gram-positive, rod-shaped, aerobic, and facultative anaerobic, motile, beta hemolytic bacterium commonly found in soil and food [1]. There are two types of toxins produce by the bacteria which are heat and acid labile enterotoxin and heat resistant emetic toxin [2].The incubation period of diarrhoeal form is 6-24 hours and it is associated with ingestion of proteinaceous foods; the shorter incubation period of emetic form is 1 - 6 hours, and is associated with consumption of farinaceous foods such as cook rice. Food poisoning attributed to Bacillus cereus have been reported and associated with cooked rice usually from Chinese restaurants and 'take-away' shops [3]. To prevent further outbreaks, it is suggested that rice should be boiled in smaller quantities on several occasions during the day and reducing the storage time before frying. After boiling the rice, it should be kept at $63^{\circ} \mathrm{C}$ or cooled quickly and transferred to a refrigerator within 2 hr. of cooking [4].

Species specification of B. Cereus [5]:

Domain: Bacteria

Phylum: Firmicute

Class: Bacilli

Order: Bacillales

Family: Bacillaceae
Genus: Bacilus

Species: B. cereus

The Bacillus cereus group consists of seven species like B.cereus, B. anthracis, B. thuringiensis, B. mycoides, B. pseudomycoides, B. weihenstephanensis and B. cytotoxicus. Bacillus food borne illnesses occur due to survival of the bacterial endospores when food is improperly cooked [5]. Cooking temperatures less than or equal to $100{ }^{\circ} \mathrm{C}\left(212^{\circ} \mathrm{F}\right)$ allow some B. cereus spores to survive [5].

\section{History of Outbreaks}

Bacillus cereus isolated from air in a cowshed by Frankland and Frankland in 1887. Many outbreaks from a variety of foods including meat and vegetable soups, cooked meat and poultry, fish, milk and ice cream were reported in Europe since 1950. In 1969, it was first well-documented in the USA. B. cereus associated vomiting type poisoning was reported in 1971 representing the nausea and vomiting 1-5 $\mathrm{h}$ after consumption of the intoxicated meal. The incubation period may vary from 15-30 minutes or 6-12 hours. It has been proved that all the vomiting type outbreaks were associated with consumption of cooked rice. This type of poisoning resembles with staphylococcal food poisoning [6].

\section{Toxins of B. Cereus}

B. cereus is producing one emetic toxin (ETE) and three different enterotoxins. Three pore-forming enterotoxins responsible for the diarrhoeal type of food poisoning are Hemolysin BL ( $\mathrm{Hbl}$ ), Non- 
haemolytic enterotoxin (Nhe), and Cytotoxin K (CytK). Hbl and Nhe each consist of three different protein components, named L2, L1, and B, and NheA, NheB and NheC, respectively, while CytK is a single-component toxin [7].

\section{Factors affecting growth of $B$. cereus}

Conducive temperature for $\mathrm{B}$. Cereus ranges from $7-49^{\circ} \mathrm{C}(44.6$ $\left.120.2^{\circ} \mathrm{F}\right)$ with a minimum of $4-5^{\circ} \mathrm{C}\left(39.2-41^{\circ} \mathrm{F}\right)$ and a maximum around $48-50^{\circ} \mathrm{C}\left(118.4-122^{\circ} \mathrm{F}\right)$. Spore germination temperature range from $8-30^{\circ} \mathrm{C}\left(46.4-86^{\circ} \mathrm{F}\right)$, $\mathrm{pH}$ range for growth $\mathrm{pH}$ 4.9-9.3, Minimum water activity $0.91-0.93$, Salt concentration as high as $7.5 \% \mathrm{NaCl}$. Thermal D value for spores at $100^{\circ} \mathrm{C}$ around $3 \mathrm{~min}$, but some spores much more resistant. Spores are more resistant to irradiation than vegetative cells. The dose for $90 \%$ reduction of spores is 1.25 - 4kGy, and 0.17-0.65 kGy of vegetative cells [6].

\section{Studies Revealed the Bacillus Cereus is associated with Fried Rice Syndrome}

One study conducted for identify the exposure pathway of fried rice dishes and evaluation of its microbiological quality from Chinese restaurants. Exposure pathway for fried rice was assessed in terms of time, temperature, and serving size by phases from preparation to consumer consumption. The microbiological quality of 32 samples was evaluated for the levels of Bacillus cereus, aerobic mesophilic plate count (APC), and coliforms. One serving size of fried rice dishes was $352.2 \mathrm{~g}$. The final temperature of fried rice dishes at the consumption point was $66.1^{\circ} \mathrm{C}$ for cook-to order restaurants, and $59.8^{\circ} \mathrm{C}$ for reheat-to-cook type restaurants. The prevalence of B. cereus detected in cooked rice at consumption point was $37.5 \%$. Production types, final temperature at cooking, and consumption phases were associated with contamination level of $B$. cereus $(\mathrm{p}<0.05)[8]$.

A case control study suggested that Bacillus cereus food poisoning is a short incubation period, characterized predominantly by sudden onset of nausea and vomiting in some cases and in others by abdominal colic, severe watery diarrhoea and tenesmus. The illness generally persists no longer than 24 hours and is rarely fatal. In February 1996 the Department of Public Health investigated an outbreak of food poisoning involving at least 92 persons among local and foreign guests at a local hotel. B. cereus was implicated as a cause of the outbreak. The study was performed on 61 cases and 80 controls from among hotel residents using a detailed questionnaire. Consumption of rice at a hotel lunch was associated with subsequent development of symptoms (OR $=2.97,95 \% \mathrm{Cl} 1.34$ -6.77). The food-specific attack rate for rice was $0.53(\mathrm{P}=0.0034)$. $\mathrm{B}$. cereus ( $7.5 \times 103$ organisms /g) was isolated from leftover samples of boiled rice and from the stools of three patients [9].

\section{Clinical Features of Fried Rice Syndrome}

The emetic form presents with vomiting, nausea, abdominal pain and occasionally late onset diarrhoea which can resemble
S. aureus food poisoning in its symptoms and incubation period. This is usually mild, lasting less than 12 hours. The diarrhoeal form usually presents with abdominal pain, diarrhoea (often watery and profuse) and tenesmus occasionally followed by mild nausea and diarrhoea. Symptoms generally subside after 24 hours. The diarrhoeal form may be difficult to distinguish from Clostridium perfringens foodborne intoxication [10].

\section{Diagnosis of FRS:}

1. PCR and immunohistochemistry.

2. CNS tissue examination

3. Cerebrospinal fluid (CSF) analysis

4. Multilocus sequence typing (MST)

5. B. cereus biofilms

6. Transcriptome analyses by RNA sequencing (RNA-seq)

\section{Preventive measures of Fried Rice Syndrome}

1. Processing (cooked thoroughly and cooled rapidly) is one of the easiest ways to prevent foodborne illness associated with Bacillus spp.

2. Cooked foods should be kept at temperature of $145 \mathrm{oF}$ or above for a minimum of 15 seconds (2001 Food Code).

3. Hot food should be kept at a temperature of $140 \mathrm{oF}$ or higher.

4. Reheating cooked food should be kept to $165 \mathrm{oF}$.

5. Frozen food should remain frozen until it is used.

6. If frozen food is displayed in a refrigerated case and allowed to thaw, the food should remain at $410 \mathrm{o}$ or below.

7. All food operators and consumers should understand the roles of food safety throughout the food chain by incorporating the policy development at the national and international levels.

8. It is needed to address risks of FBDs and ensure food safety. Education and training are needed on prevention of FBDs among food producers, suppliers, handlers and the general public, including women and school children.

9. Steaming under pressure, roasting, frying and grilling foods can destroy the vegetative cells and spores (11).

10. Foods infested with the diarrheal toxin can be inactivated by heating for $5 \mathrm{~min}$ at $133^{\circ} \mathrm{F}$ [11].

11. Foods infested with the emetic toxin need to be heated to $259^{\circ} \mathrm{F}$ for more than $90 \mathrm{~min}$. Reheating foods until they are steaming is not enough to kill the emetic toxin [11].

\section{References}

1. Edward J Bottone (2010) Bacillus cereus, a Volatile Human Pathogen. Clin Microbiol Rev 23(2): 382-398. 
2. Anita Tewari, Swaid Abdullah (2015) Bacillus cereus food poisoning: international and Indian perspective. J Food Sci Technol 52(5): 25002511.

3. R J Gilbert, M F Stringer, T C Peace (1974) The survival and growth of Bacillus cereus in boiled and fried rice in relation to outbreaks of food poisoning; J Hyg (Lond) 73(3): 433-444.

4. Gilbert RJ, Stringer MF, Peace TC (1974) The survival and growth of Bacillus cereus in boiled and fried rice in relation to outbreaks of food poisoning. J Hyg (Lond) 73(3): 433-44.

5. https://en.wikipedia.org/wiki/Bacillus_cereus002E

6. Mehrdad Tajkarimi (2007) Bacillus cereus. Pp. 250H6-1-250H6-6.

7. Anita Tewari, Swaid Abdullah (2015) Bacillus cereus food poisoning: international and Indian perspective. J Food Sci Technol 52(5): 25002511.

8. Hye ja Chang, Ji hye Lee, Bo ra Han, Tongkyung Kwak, Jun Kim (2011) Prevalence of the levels of Bacillus cereus in fried rice dishes and its exposure assessment from Chinese-style restaurants. Food Science and Biotechnology 20: 1351.

9. K A Vella (1997) A case-control study investigating an outbreak of Bacillus cereus food poisoning in a hotel in Malta, Maltese Medical 23 Journal 9(1): 23-27.

10. (2012) Bacillus cereus food-borneillness.

11. (2004) National Institutes of Health (NIH), the National Institute of Allergy and Infectious Diseases (NIAID), and the National Food Processors Association (NFPA). 